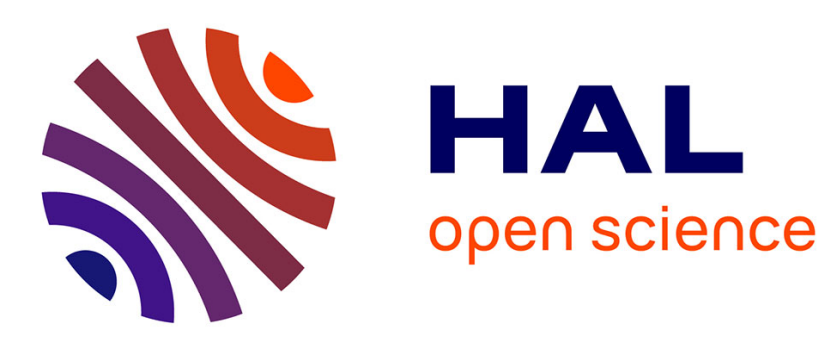

\title{
Sur l'application de la théorie de Compton au rayonnement $\beta$ et $\gamma$ des corps radioactifs
}

\author{
Pierre Curie
}

\section{To cite this version:}

Pierre Curie. Sur l'application de la théorie de Compton au rayonnement $\beta$ et $\gamma$ des corps radioactifs. J. Phys. Radium, 1926, 7 (4), pp.97-108. 10.1051/jphysrad:019260070409700 . jpa-00205247

\section{HAL Id: jpa-00205247 https://hal.science/jpa-00205247}

Submitted on 1 Jan 1926

HAL is a multi-disciplinary open access archive for the deposit and dissemination of scientific research documents, whether they are published or not. The documents may come from teaching and research institutions in France or abroad, or from public or private research centers.
L'archive ouverte pluridisciplinaire HAL, est destinée au dépôt et à la diffusion de documents scientifiques de niveau recherche, publiés ou non, émanant des établissements d'enseignement et de recherche français ou étrangers, des laboratoires publics ou privés. 


\title{
LE JOURNAL DE PHYSIQUE
}

\section{LE RADIUM}

\author{
SUR L'APPLIGATION DE LA THÉORIE DE COMPTON \\ AU RAYONNEMENT $\beta$ ET $\%$ DES CORPS RADIOACTIFS \\ par Mme P. CURIE.
}

\begin{abstract}
Sommaire. - Dans ce mémoire, on a examiné comment la théorie proposée par Compton pour la diffusion de quanta sur les électrons libres peut s'appliquer à un groupe monochromatique de rayons $\gamma$ émis par un radioélément, si l'on prévoit leur diffusion, soit sur les électrons de l'atome émetteur,' soit sur ceux d'atomes étrangers voisins, cette diffusion étant accompagnée de l'émission correspondante d'électrons dits de recul ou de choc. On a examiné la répartition d'énergie pour les rayons $\gamma$ diffusés et pour les électrons de choc ainsi que d'autres répartitions qui onit un rapport direct avec l'expérience (répartition des longueurs d'onde pour les rayons $\gamma$, répartition des quantités de mouvement et de leurs inverses pour les rayons $\beta$ ). Les formules et les courbes données peuvent servir pour la discussion des résultats obtenus dans l'élude de la composition du rayonnement $\gamma$ et $\beta$ primaire et secondlaire des radioéléments.
\end{abstract}

1. La théorie de Compton. - Un des problèmes les plus importants de la physique moderne est l'étude des conditions dans lesquelles a lieu l'échange d'énergie entre les électrons et le rayonnement électromagnétique, c'est-à-dire la conversion de l'énergie cinétique d'un électron en énergie de radiation électromagnétique ainsi que le phénomène réciproque où la radiation électromagnétique agit sur un électron pour l'extraire de l'atome et lui communiquer une énergie cinétique. D'après la loi d'équivalence photoélectrique d'Einstein, un électron occupant dans l'atome un niveau d'énergie $K, L, M$, etc..., peut emprunter à la radiation incidente de fréquence $\nu$ un quantum d'énergie $h \nu$ qui, intégralement absorbé, est utilisé en partie pour fournir lé travail de l'extraction de l'électron de l'atome $w_{i}=w_{\mathrm{K}}, w_{\mathrm{L}}$, etc..., tandis que l'excédent est acquis à l'électron sous forme d'énergie cinétique $W$, selon l'équation

$$
h \nu=w_{i}+W, \quad \text { avec } \quad W=m c^{2}\left[\frac{1}{\sqrt{1-\beta^{2}}}-1\right]
$$

où $m$ est la masse de repos de l'électron; $c$, la vitesse de la lumière, et $\beta c$, celle de l'électron. On peut mesurer $\beta$ pour des rayons corpusculaires produits en recevant sur un écran matériel des rayons $X$ de fréquence connue ; il suffit, pour cela, d'obtenir le spectre magnétique de ces rayons $\beta$ secondaires, nommés aussi photoélectrons. De nombreuses expériences ont prouvé que l'émission de photoélectrons selon la loi d'Einstein est un phénomène régulièrement observé sur différents radiateurs $\left({ }^{1}\right)$.

Plus récemment, A.-H. Compton a proposé une théorie d'échange d'énergie et de quantité de mouvement par choc entre un électron et un quantum ${ }^{2}{ }^{2}$. L'électron est considéré

(1) M. dz Brogla, Les rayons X, Conférences-Rapports, t. 1, Paris.

(2) A.-H. Conptox, Phys. Rev., t. 21 (1923), p. 483. - Debye, Physik. Zts., t. 24 (1923), p. 161.

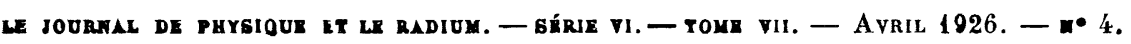


en repos et libre de toute attache. Le quantum est considéré comme un grain dénergie de valeur $h v$ qui suit, avec la vitesse $c$, une trajectoire rectiligne et possède une quantité de mouvement $h_{v} / c$. Lors du choc, une partie de l'énergie et de la quantité de mouvement est cédée à l'électron de recul ou de choc qui prend la vitesse $\beta c$, la quantité de mouvement $m \beta c\left(1-\beta^{-1 / 2}\right.$ et l'énergie $W$, tandis que le quantum résiduel diffusé a une fréquence $\nu_{\theta}$ inférieure à la fréquence $\nu_{0}$ du quantum incident. En écrivant qu'il y a conservation de l'énergie et de la quantité de mouvement, on obtient trois équations entre les variables $\nu_{0}, v_{\theta}, \beta, \theta$ et $\varphi, \theta$ et $\varphi$ étant respectivement les angles que font, avec la direction du quantum incident, celles du quantum diffusé et de l'électron de choc (fig. 1). Connaissant

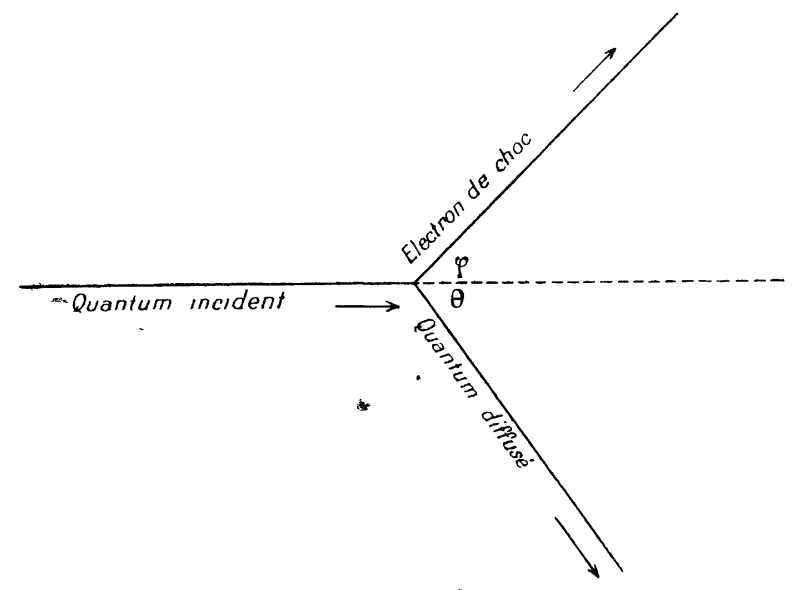

Fig. 1 .

$\nu_{0}$, on peut, à l'aide de ces équations, déterminer $\nu_{\theta}, \beta$ et $\varphi_{i}$ en fonetion de $\theta$. Introduisant, avec Compton, un paramètre $\alpha=\frac{h \nu_{0}}{m c^{2}}$, on trouve :

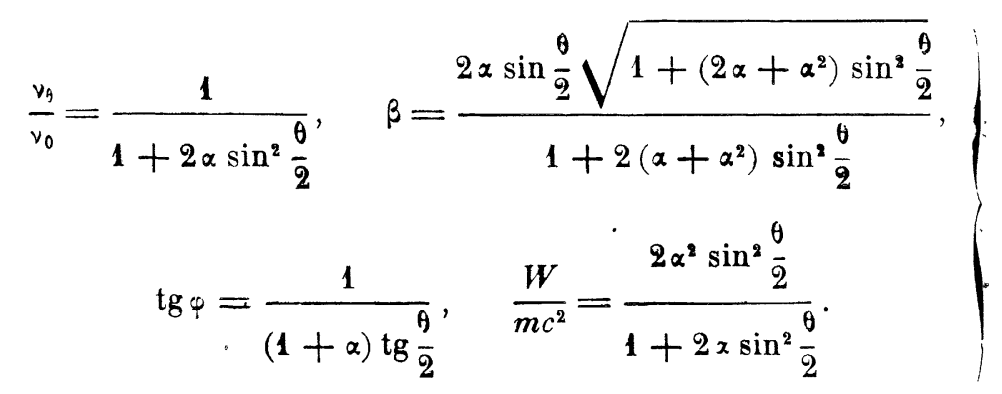

Le paramètre $\alpha$ est le rapport de la fréquence primitive $\nu_{0}$ à une fréquence fondamentale $m c^{2} / h$ dont la valeur numérique est $1,24 \times 10^{20}$ et le potentiel équivalent 509000 volts. C'est aussi le rapport de l'énergie $h v_{0}$ à celle de l'électron au repos. Ta dis que l'angle $\theta$ augmente de 0 à $\pi$, la fréquence du quantum diffusé passe de $\nu_{0} \grave{a} \frac{\nu_{0}}{1+2 \alpha}$, et la longueur d'onde, de $\lambda_{0}$ à $\lambda_{0}(1+2 \alpha) ;$ l'accroissement $2 \alpha \lambda_{0}$ étant égal à

$$
\frac{2 h \nu_{0}}{m c^{2}} \times \frac{c}{\nu_{0}}=2 \frac{h}{m c},
$$

est indépendant de $\lambda_{o}$. Les électrons sont émis vers l'avant par rapport à la direction du quantum incident, $p$ restant compris entre 0 et $\pi / 2$, tandis que la vitesse et l'énergie, nulles 
quand $\theta=0, \varphi=\pi, 2$, prennent leurs valeurs maxima pour $\theta=\pi, \varphi=0$. Ces valeurs sont (électron projeté dans la direction du quantum incident)

$$
\beta_{\max }=\frac{2 \alpha(1+\alpha)}{1+2 \approx+2 \alpha^{2}}, \quad W_{\max }=h v_{0} \times \frac{2 \alpha}{1+2 \alpha} .
$$

L'électron de choc ne peut, dans cette manière de voir, absorber intégralement l'énergio du quantum incident; il en absorbe une fraction qui est négligeable pour une radiation de basse fréquence ( $\alpha$ petit) et tend vers 1 quand la fréquence augmente. Cependant, pour $\alpha=1$, c'est-à-dire pour une radiation à potentiel équivalent élevé ( 509000 volts), l'énergie absorbée par l'électron n'est encore que $\mathbf{2} / 3 \mathrm{du}$ quantum incident. II y a donc une différence fondamentale entre ce mode de conversion de l'énergie et celui qui est applicable à l'émission de photoélectrons, puisque, chez ces derniers, lequantum incident se retrouve entièrement à l'état d'énergie cinétique quand l'électron est extrait d'un niveau périphérique de l'atome et que le travail d'extraction est négligeable. Les conditions qui déterminent le choix entre les deux modes de conversion restent obscures.

La théorie de Compton a été l'objet de vérifications expérimentales qui lui sont farorables. Le changement de longueur d'onde des rayons $X$ par diffusion sur cortains corps légers (graphite, paraffine), a été observé aussi bien par l'auteur de la théorie (') que par d'autres savants. Utilisant comme radiation primaire la raie $\mathrm{K}$ du molybdène et analysant par diffraction la radiation diffusée sous différents angles, on a trouvé qu'elle se compose d'une raie non modifiée et d'une raie modifiée selon les prévisions de la théorie. La présence de la raie non modifiée prouve qu'aux processus de diffusion quantique se superpose un autre qui ne comporte pas de réduction de fréquence. C'est ce dernier qui assurerait les conditions qui rendent possible la spectrographie des rayons $\mathrm{X}\left({ }^{2}\right)$.

Une autre vérification de la théorie de Compton concerne la mesure du coefficient le diffusion des rayons $\boldsymbol{X}$ et des rayons $\gamma$. On sait que le coefficient d'absorption massique global de ces rayons, tel qu'il est mesuré par une chambre d'ionisation avec écrans interposés, se compose d'un terme d'absorption vraie proportionnel à $\lambda^{3}$ ( $\lambda$, longueur d'onde incidente) et à $N^{3}$ ( $N$, nombre atomique de l'élément absorbant), et d'un terme de diffusion. L'absorption vraie correspond, croit-on, à l'émission de photoélectrons. La diffusion est une réémission de rayonnement électromagnétique secondaire dans toutes les directions autour d'un faisceau primaire canalisé ; l'énergie ainsi soustraite à celui-ci peut être mesurée à l'aide de dispositifs appropriés. J.-J. Thomson a donné, pour le coefficient de diffusion massique $\sigma / \rho$, une évaluation basée sur la théorie classique du rayonnement, en bon accord avec les expériences relatives aux rayons $X$ de fréquence modérée. Ce coefficient est alors approximativement égal à 0,2 , quelle que soit la longueur d'onde et la nature du radiateur : la diffusion a lieu symétriquement vers l'avant et l'arrière. Cependant, pour les rayons $\gamma$ du radium $\mathrm{C}$ il n'en est plus de même. I e coefficient de diffusion est beaucoup plus petit que 0,2 et la diffusion a lieu vers l'avant bien plus que vers l'arrière. Pour calculer l'énergie soustraite par diffusion au faisceau primitif canalisé, Compton a cherché une loi de distribution des quanta diffusés autour de la direction du quantum incident. La formule de probabilité qu'il a établie peut paraître arbitraire ; elle est cependant rendue plausible par le principe dont elle s'inspire d'établir la continuité entre le coefficient cherché et le coefficient de J.-J. Thomson qui reste valable pour les rayons $\mathrm{X}$ usuels. La proportion des quanta diffusés par les électrons libres dans l'angle solide compris entre $\theta$ et $\theta+d \theta$ est admise égale à $P_{\theta} \mathrm{d} \theta$ tel que

$$
P_{\theta} \mathrm{d}_{\theta}=\frac{3}{8}(1+2 \alpha) \sin \theta \frac{\left(1+2 \alpha+2 \alpha^{2}\right)\left(1+\cos ^{2} \theta\right)-4 \alpha(1+\alpha) \cos \theta}{(1+\alpha-\alpha \cos \theta)^{4}} \mathrm{~d} \theta .
$$

Admettant, de plus, que dans la direction $\theta=0$, où le quantum n'est pas modifié, la fraction

(') A.-H. Compton, Phys. Rev., t. 22 (1923), p. 409. - M. nв Broglir et A. Daurillibr, J. Phys., t. 6 (192̈), p. 369.

(2) Jaurcer, Phys. Rev., t. 25 (1925), p. 314, a fait une tentative pour expliquer la présence de la raie modifiée et de la raie non modifiée par la diffusion de quanta sur des électrons liés à l'atome. Quand l'énergie fournie à l'électron est inférieure à l'énergie de liaison, on suppose que le choc est sans effet. 
diffusée est conforme à la théorie classique, on trouve que le coefficient de diffusion $\sigma$ se déduit du coefficient $\sigma_{0}$ qui correspond à la théorie de J.-J. Thomson, par la relation

$$
\sigma=\frac{\sigma_{0}}{1+2 x}=\sigma_{s}+\sigma_{a} \text {. }
$$

L'énergie ainsi soustraite au faisceau se compose de deux parties : l'énergie des quanta diffusés et celle des électrons de choc, respectivement caractérisées par les coefficients

$$
\sigma_{s}=\frac{1+a}{(1+2 \alpha)^{2}} \sigma_{0}, \quad \sigma_{a}=\frac{\alpha}{(1+2 x)^{2}} \sigma_{0}, \quad \frac{\sigma_{a}}{\sigma_{s}}=\frac{a}{1+\alpha} .
$$

L'énergie des électrons dechoc, négligeable par rapport à celle des quanta diffusés quand $\alpha$ est petit, tend à devenir égale à cette dernière pour les valeurs élevées de $\alpha$.

Les prévisions de cette théorie sont en bon accord avec les expériences sur la diffusion des rayons $\gamma$ du RaC. D'autre part, les électrons de choc ont été observés par la méthode des trajectoires de brouillard de C.-T.-R. Wilson ; et ces observations sont aussi conformes aux prévisions. Cependant, il existe encore très peu de documentation expérimentale numérique sur ces électrons de choc dont les vitesses sont généralement faibles ( $\left.{ }^{1}\right)$.

Ainsi, il y aurait trois modes d'action des quanta sur les électrons : 1. le quantum est diffusé sans changement de fréquence ; 2 . le quantum est absorbé et cède son énergie à un atome ; 3 . le quantum partage son énergie avec un électron.

2. Composition du rayonnement $\beta$ et $\gamma$ des radioéléments. - Le rayonnoment $\beta$ et le rayonnement $\gamma$ d'un radioélément peuvent être étudiés à l'aide de mesures de coefficients d'absorption et de l'analyse spectrale. Pour les rayons $\gamma$, on étudie le spectre de diffraction ; pour les rayons $\beta$, le spectre magnétique. On obtient, en général, un spectre continu etdes raies correspondant à des fréquences déterminées pour les rayons $\gamma$, à des vitesses déterminées pour les rayons $\beta$. Le spectre continu prend la forme de bandes plus ou moins étendues.

De nombreux travaux ont mis en évidence les relations qui existent entre les rayons $\gamma$ et les rayons $\beta$. Il a été reconnu que, parmi les raies du spectre magnétique des rayons $\beta$, la plupart sont dues à des photoélectrons émis des niveaux $\mathrm{K}, \mathrm{L}$, etc., de l'atome radioactif par l'action de rayons $\gamma$ monochromatiques émis du noyau de ce même atome $\left({ }^{2}\right)$.

Par contre, l'interprétation du spectre continu paraît moins facile. Partant de l'idée que les rayons $\beta$ primaires et les rayons $\gamma$ nucléaires correspondent à des groupes homogènes, certains auteurs ont cherché dans l'effet Compton l'origine du spectre continu et des bandes, au total ou en partie $\left({ }^{8}\right)$.

Il paraît donc désirable de se rendre compte de ce que l'on peut attendre de l'effet Compton obtenu à partir de groupes de rayons $\gamma$ monochromatiques agissant sur les électrons qui entourent le noyau de l'atome. La théorie de Compton s'applique aux électrons dits a libres ", c'est-à-dire à oeux dont la liaison est faible. De tels électrons existent en grand nombre à la périphérie des atomes lourds comme ceux des radioéléments. L'examen des propriétés optiques et des potentiels d'ionisation prouve que le degré de liaison est du même ordre dans la région périphérique de tous les atomes.

3. Conversion de rayons $\gamma$ en rayons $\beta$ par effet Compton. - La conversion d'un groupe monochromatique de rayons $\gamma$ par effet Compton est partielle. Elle doit donner, en principe, une bande de rayons $\gamma$ diffusés limitée du côté des basses et des hautes fréquences et une bande de rayons $\beta$ limitée du côté des grandes vitesses seulement. J'admettrai que l'expérimentation a lieu sur une couche infiniment mince de matière radioactive, de sorte que, la conversion ayant été faite dans l'atome radioactif dont sont originaires les rayons $\%$, les rayons $\beta$ et $\gamma$ qui en résultent ne sont plus modifiés par un passage ultérieur au travers

(1) Dans un travail récent, Hoffmann [Zts. f. Phys., t. 36 (1926), p. 251] a vérifié que le coefficient d'absorption des rayons $\gamma$ de RaC, diffusés sous différents angles sur le carbone, varie conformément aux prévisions de la théorie.

(2) Elus, Proc. Roy. Soc., t. 99 (1921), p. 261 ; t. 101 (1922), p. 1. - Rlurs et Srinrre, Proc. Roy. Soc., t. 105 (1924), p. 165 et 185. - L. Mritrer, Zts. f. Phys., t. 9 (1922), p. 131 et 145; t. 11 (1922), p. 35. J. ThiBaud, Thèse de Doctorat, Paris, (1925).

(3) L. Mutnar, Zts. f. Phys., t. 19 (1923), p. 307. 
de la matière. J'admettrai aussi que les rayons $\gamma$ monochromatiques sur lesquels porte la conversion, sont émis indifféremment dans toutes les directions. En ce cas, la formule de probabilité qui détermine la proportion $\boldsymbol{P}_{\theta} \mathrm{d} \theta$ de quanta ayant la valeur $\nu_{\theta}$ est également valable pour toute direction d'émission. A chacun de ces quanta correspond un électron de choc dont l'énergie est connue en fonction de $\theta$.

Le mème raisonnement s'applique aux rayons $\$$ secondaires issus d'une source radioactive de petites dimensions entourée par un écran matériel très mince dont les atomes diffusent les rayons $\gamma$ primaires. Le rendement de la conversion pourraït être, en ce cas, très différent, mais la distribution des vitesses entre les électrons de choc devrait être d'autant plus semblable à celle que prévoit la théorie que l'écran diffuseur serait plus mince.

Au lieu de construire la courbe $P_{\theta}=\mathrm{f}(\theta)$ qui ne se prête pas à la discussion, on peut avoir recours à des variables dont la liaison avec le problème est plus évidente. Si $x$ est une telle variable, on peut former l'expression $\boldsymbol{P}_{x} \mathrm{~d} x=\boldsymbol{P}_{\theta} \mathrm{d} \theta$ et construire la courbe $\boldsymbol{P}_{x}$ en fonction de $x$. On devra avoir

$$
\int P_{x} \mathrm{~d} x=\int_{0}^{\pi} P_{\theta} \mathrm{d} \theta=1
$$

les limites de la première intégrale correspondant aux limites de la seconde. Si $x$ décroît quand $\theta$ croît, le calcul donne $P_{x}<0$, mais comme ce signe s'applique au sens décroissant de $x$, le signe + conviendra en tout cas au sens croissant:

Puisque, pour toutes les courbes obtenues avec"des valeurs différentes de la fréquence primaire $v_{o}$, l'aire contenue entre la courbe et les axes dans les limites prévues est la même, ces courbes donnent uniquement la répartition des quanta diffusés ou des électrons de choc en fonction des variables choisies. Quant au nombre de quanta diffusés et d'électrons de choc émis par quantum primaire incident, il serait à présumer, d'après les hypothèses de Compton (formule õ), que ce nombre varie en raison inverse de $(1+2 \alpha)$ et diminue, par conséquent, quand la fréquence primaire augmente $\left({ }^{1}\right)$.

Remarquons enfin qu'il y a avantage à employer comme unités certaines grandeurs qui s'introduisent dans les calculs; ainsi, l'énergie de repos $m c^{2}$ d'un électron peut servir comme unité d'énergie; la quantité $m c^{2} / h$ est une unité de fréquence; $m c^{2} / e$, une unité de potentiel; $h / m c$, une unité de longueur, etc.

4. Rayons $\gamma$ diffusés. - La variable la plus indiquée est, en ce cas, la longueur d'onde $\lambda$, car les distances comptées depuis la raie centrale dans un spectre de diffraction étant approximativement proportionnelles aux longueurs d'onde, la courbe $P_{\lambda}=\mathrm{f}(\lambda)$ représente approximativement ce que nous pourrions observer sur le spectre de diffractíon, si le noircissement de la plaque en chaque point était proportionnel au nombre de rayons qu'elle reçoit par unité de largeur du spectre $\left(^{2}\right)$. C'est ce nombre qui est donné, en effet, par $P_{\imath}$, que l'on calcule à l'aide des relations (2) et (4) par la formule

On a

$$
P_{\mathrm{i}}=P_{\theta} \frac{\mathrm{d} \theta}{\mathrm{d} \lambda} \text {. }
$$

$$
\lambda=\lambda_{0}\left(1+2 x \sin ^{2} \frac{\theta}{2}\right), \quad \frac{\mathrm{d} \lambda}{\mathrm{d} \theta}=\alpha \lambda_{0} \sin \theta, \quad \cos \theta=\frac{\alpha \lambda_{0}+\lambda_{0}-\lambda}{\alpha \alpha_{0}} .
$$

Substituant ces valeurs, on trouve

$$
P_{\lambda}=\frac{3}{8} \frac{(1+2 \alpha)}{\alpha^{2}} \lambda_{0} \frac{\lambda_{0}^{2}(1+2 \alpha)^{2}-2 \lambda_{0}(1+\alpha)(1+2 \alpha) \lambda+\cdot\left(1+2 \alpha+2 \alpha^{2}\right) \lambda^{2}}{\lambda^{1}} .
$$

Les limites de variations pour $\lambda_{\text {sont }} \lambda_{0}$ et $\lambda_{0}(1+2 \alpha)$, dont la différence

$$
2 \alpha \lambda_{0}=\frac{2 h}{m c}
$$

(') On peut Gependant prévoir, pour les fréquences primaires modérées, un accroissement de l'effet Compton avec la fréqnence, provenant de ce qu'à mesure que celle-ci augmente. de nouveaux groupes d'électrons à liaison de plus en plus rigide commencent à participer au phénomène.

() En réalité, le noircissement, à nombre de rayons égal, est une fonction de la longueur d'onde. 
est indépendante de $\lambda_{0}$, de sorte qu'à chaque groupe monochromatique de rayons $\gamma$ correspond, dans le même spectre de diffraction, une bande de même largeur débutant à la raie primaire et occupant un intervalle de longueur d'onde de 4,86.10-10 $\mathrm{cm}$ ou 0,0486 unités $\mathrm{i}$. Il s'agit donc ici de bandes étroites mais accessibles à la mesure. On peut voir que si $a>0,547$, l'intensité dans la bande décroît constamment depuis $\lambda_{0}$ jusqu'à $\lambda_{0}(1+2 \approx)$. tandis que si $x<0,5$, il existe un minimum d'intensité à l'intérieur de la bande, le bord le plus important étant toujours celui qui touche à la raie primaire.

- Si l'on remplace la variable $\lambda$ par la variable « réduite $\xi \xi=\frac{\lambda}{\alpha \lambda_{0}}$, ce qui revient à prendre comme unité de longueur $h / m c$, on ob tient la relation

$$
P_{\xi}=\frac{3}{8} \frac{1+2 \alpha}{\alpha^{8}} \cdot \frac{(1+2 \alpha)^{2}-2 \alpha(1+\alpha)(1+2 \alpha) \xi+\alpha^{2}\left(1+2 \alpha+2 \alpha^{2}\right) \xi^{2}}{\xi^{4}} .
$$

Les courbes de la figure 2 représentent cette relation pour $\alpha=1$ et $\alpha=0,1$ (rayons primaires correspondant respectivement à $ّ 09$ et $50,9 \mathrm{kv}$.

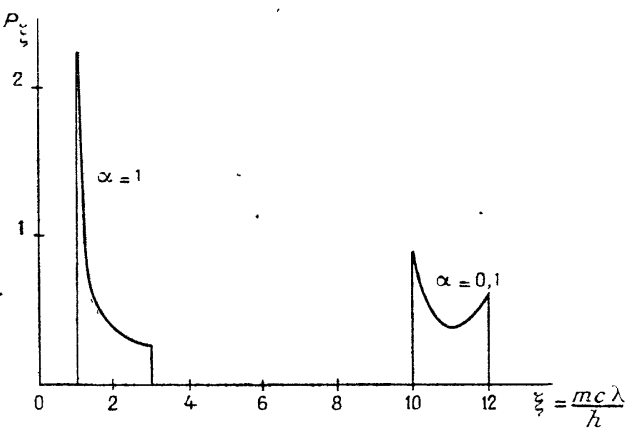

Fig. 2 .

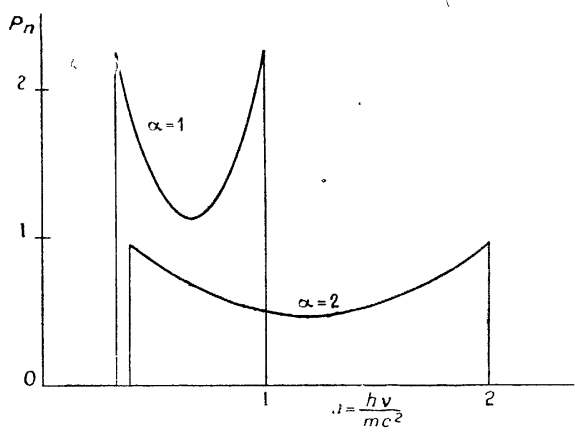

Fig. 3 .

Si l'on envisage la distribution des fréquences $v$, ou, ce qui revient au même, celle des énergies $h v$ des quanta diffusés, on trouve une loi parabolique simple

$$
P_{y}=\frac{3}{8} \frac{1+2 \alpha}{\alpha^{3} \nu_{0}^{2}}\left[\left(1+2 \alpha+2 \alpha^{2}\right) \nu_{0}^{2}-2(1+\alpha)(1+2 \alpha) \nu_{0} \nu+(1+2 \alpha)^{2} \nu^{2}\right],
$$

ou, en posant

$$
\begin{gathered}
n=\frac{\alpha \nu}{\nu_{0}}=\frac{h \nu}{m c^{2}} \\
P_{n}=\frac{3}{8} \frac{1+2 \alpha}{\alpha^{6}}\left[\alpha^{2}\left(1+2 \alpha+2 \alpha^{2}\right)-2 \alpha(1+\alpha)(1+2 \alpha) n+(1+2 \alpha)^{2} n^{2}\right] .
\end{gathered}
$$

C'est une distribution symétrique avec valeurs égales de la probabilité aux deux limites qui sont

$$
v_{0} \text { et } \frac{v_{0}}{1+2 \alpha} \text { pour } \nu, \quad \alpha \text { et } \frac{\alpha}{1+2 \alpha} \text { pour } n,
$$

et valeur moitié moindre pour la fréquence moyenne entre ces limites. La distance des limites croît avec $\alpha$. Les courbes représentatives pour $\alpha=1$ et $\alpha=2$ sont données dans la figure 3.

5. Electrons de choc. - Commençons par examiner la distribution de leur énergie $W$ dont les valeurs limites, d'après ( 2 ), sont

$$
W=0 \quad \text { et } \quad W=\frac{2 \dot{\alpha}^{2}}{1+2 \alpha} m c^{2} .
$$


On peut prendre comme variable réduite

d'où

$$
s=\frac{W}{m c^{2}}=\frac{2 \alpha^{2} \sin ^{2} \frac{\theta}{2}}{1+2 \alpha \sin ^{2} \frac{\theta}{2}}
$$

$$
\begin{gathered}
\sin ^{2} \frac{\theta}{2}=\frac{s}{2 \alpha(\alpha-s)}, \quad \cos \theta=\frac{\alpha^{2}-s-\alpha s}{\alpha(\alpha-s)}, \quad \frac{\mathrm{d} \theta}{\mathrm{d} s}=\frac{\left(1+2 \alpha \sin ^{2} \frac{\theta}{2}\right)^{2}}{\alpha^{2} \sin \theta} \\
P_{s}=\frac{3}{8} \frac{1+2 \alpha}{\alpha^{6}}\left[2 \alpha^{4}-2 \alpha^{2}(1+2 \alpha) s+(1+2 \alpha)^{2} s^{2}\right] .
\end{gathered}
$$

La courbe qui représente $P_{\text {s }}$ est un arc de parabole (fig. 4) ayant même ordonnée pour $s=0$ et pour la valeur limite $s=S ;$ pour $s=\frac{s}{2}, P_{s}$ passe par un minimum dontl'ordonnée est la moitié de chaque ordonnée limite. Ainsi, l'énergie limite correspondant, par exemple, à 1000000 volts, le nombre de rayons $\beta$ secondaires d'énergies comprises entre 0 et 500000 volts serait le même que celui des rayons dont les énergies seraient comprises entre 500000 volts et 1000000 volts. L'étendue du domaine d'énergie représenté croìt constamment avec $\alpha$, tandis que les ordonnées limites décroissent dans le même rapport, comme dans la figure 4, l'aire com prise entre la courbe, les ordonnées limites et l'axe des $s$ devant rester constante.

Considérons, maintenant, au lieu de l'énergie, la quantité de mouvement d'un électron de choc. Cette donnée est reliée au rayon de courbure $R$ d'une trajectoire cir-

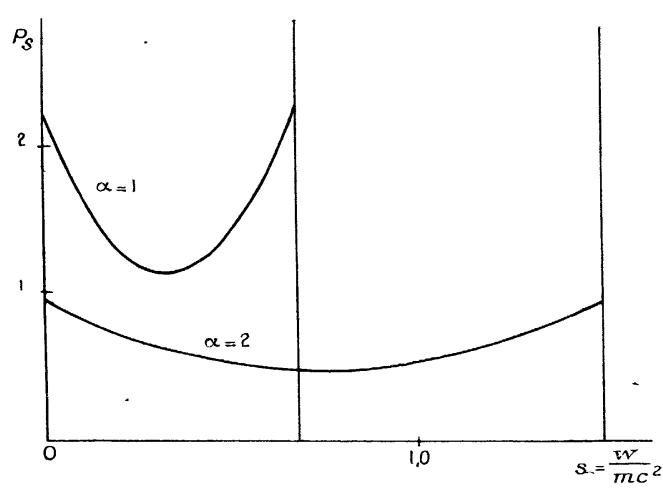

Fig. 4 . culaire que décrit l'électron dans un champ magnétique uniforme d'intensité $H$ perpendiculaire à sa vitesse. On a la relation

où $\boldsymbol{e}$ est la charge de l'électron.

$$
R H=\frac{m c \beta}{e \sqrt{1-\beta^{2}}}
$$

La déviation magnétique mesurée sur les clichés de spectres magnétiques est une fonction du rayon de courbure, mais cette fonction dépend du dispositif expérimental employé.

Nous examinerons successivement deux cas extrêmes avec des variables $x$ et $y$ proportionnelles respectivement à $R H$ et à son inverse; nous poserons

$$
x=\frac{R H}{g}=\frac{\beta}{\sqrt{1-\beta^{2}}}, \quad y=\frac{g}{R H}=\frac{\sqrt{1-\beta^{2}}}{\beta},
$$

où $g=\frac{m c}{e}=1696$ unités E. M., admettant

$$
\frac{e}{m}=1,77 \times 10^{7} \text { U. E. M. }
$$

Les distributions correspondantes sont aisément obtenues à partir de celle des énergies, en utilisant la relation

$$
s=\frac{1}{\sqrt{1-\beta^{2}}}-1=\sqrt{1+\left(\frac{R H}{g}\right)^{2}}-1,
$$


d'où l'on déduit

On trouve les formules

$$
\begin{gathered}
s=\sqrt{1+x^{2}}-1 \quad \frac{\mathrm{d} s}{\mathrm{~d} x}=\frac{x}{\sqrt{1+x^{2}}} \\
s=\frac{\sqrt{1+y^{2}}}{y}-1 \quad \frac{\mathrm{d} s}{\mathrm{~d} y}=-\frac{1}{y^{2} \sqrt{1+y^{2}}} .
\end{gathered}
$$

$P_{x}=\frac{3}{8} \frac{1+2 \alpha}{\alpha^{6}}\left[\left\{\alpha^{4}+(1+\alpha)^{4}\right\} \frac{x}{\sqrt{1+x^{2}}}+(1+2 \alpha)^{2} x \sqrt{1+x^{2}}-2(1+\alpha)^{2}(1+2 \alpha) x\right] ;$

valeurs limites :

$$
\begin{aligned}
s=0, & x=0, \quad P_{x}=0 \\
s=S, \quad x=\frac{2 \alpha(1+\alpha)}{1+2 x}=X, & P_{X}=\frac{3(1+2 \alpha)(1+\alpha)}{2 x\left(1+2 \alpha+2 \alpha^{2}\right)} \\
P_{y}=\frac{3}{8} \frac{1+2 \alpha}{a^{6}}\left[i \alpha^{4}+(1+\alpha)^{4}\right. & \left.\frac{1}{y^{2} \sqrt{1+y^{2}}}+\frac{(1+2 \alpha)^{2} \sqrt{1+y^{2}}}{y^{4}}-\frac{2(1+\alpha)^{2}(1+2 \alpha)}{y^{3}}\right]
\end{aligned}
$$

valeurs limites :

$$
\begin{array}{lll}
s=0, & y=\infty, & P_{y}=0 ; \\
s=S, & y=\frac{1+2 \alpha}{2 x(1+\alpha)}=Y, & P_{Y}=\frac{6 \alpha(1+\alpha)^{3}}{(1+2 x)\left(1+2 \alpha+2 \alpha^{2}\right)} .
\end{array}
$$

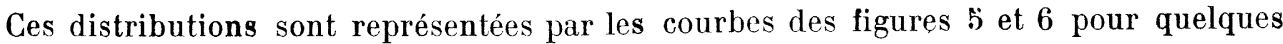
valeurs de $\alpha$. Quand $\alpha$ croît, il en est de même de l'ordonnée $P_{y}$ tandis que $P_{x}$ décroit. Les deux distributions sont très différentes. L'ordonnée maximum est, dans les deux cas, celle qui correspond à l'énergie maximum, mais, si la variable est $x$, il n'existe pas de variation très brusque; si la variable est $y$, il $\mathrm{y}$ a resserrement de rayons formant une pointe très prononcée du côté des grandes énergies. Ainsi, dans un cliché de spectre magnétique où la déviation serait proportionnelle à $x$, c'est-à-dire à $R$, à un groupe de rayons $\gamma$ monochroma-

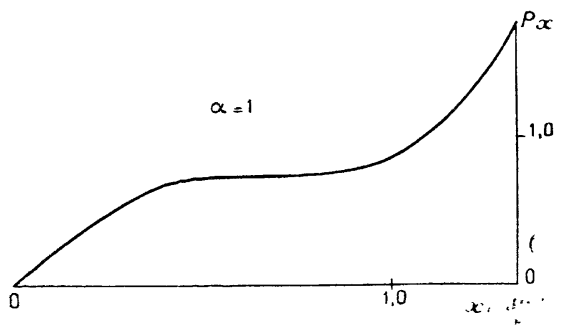

Fig. 5.

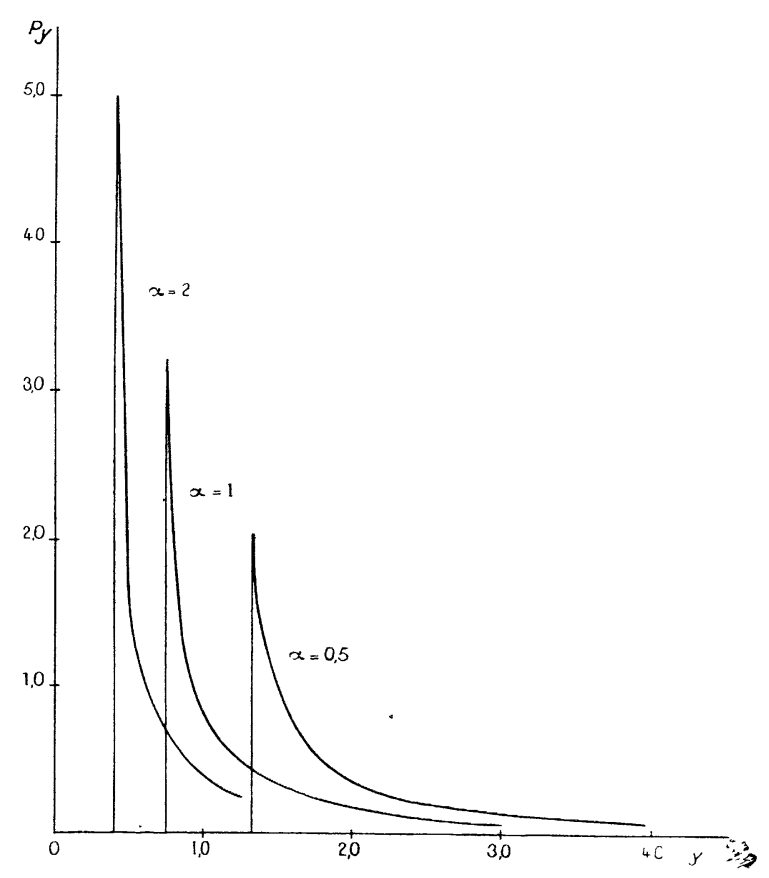

Fig. 6 .

tiques correspondrait, par effet Compton, une bande largement étalée avec densité de rayons maximum pour l'énergie maximum; si la déviation était proportionnelle à $y$, c'est-à-dire à l'inverse de $R$, à ce mème groupe correspondrait une tète de bande très accusée donnant 
l'apparence d'une raie et se terminant par une queue étalée comprenant les rayons de faible énergie.

Or, sur les clichés de spectres magnétiques, tels que les donne l'expérience, l'effet Compton pourrait précisément se traduire par les apparences ci-dessus indiquées. En effet, ces clichés sont obtenus à l'aide de deux types d'appareillage, dont l'un se rapproche de l'emploi de la variable $x$ et l'autre de l'emploi de la variable $y$. Ces types sont l'appareil $\dot{a}$ foyer et l'appareil à déviation directe; la marche des rayons dans un champ perpendiculaire au plan de la figure est représentée dans la figure 7 . Dans le premier cas, la déviation z
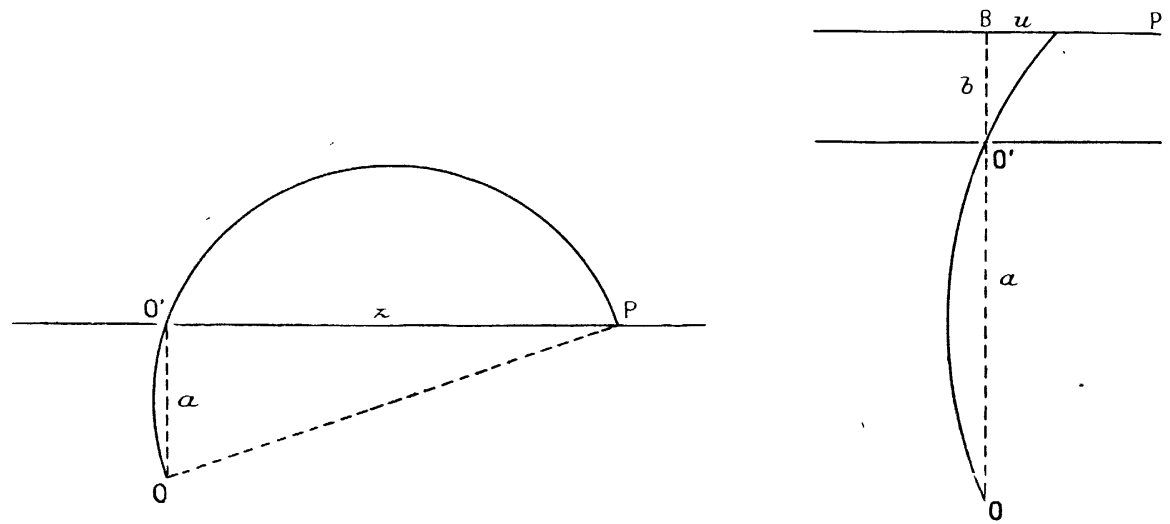

Fig. T.

correspond à un rayon défini par la source linéaire 0 , la fente diaphragme $0^{\prime}\left(00^{\prime}=a\right)$ et le rayon de courbure $R$, ayant décrit une trajectoire demi-circulaire et se terminant sur la plaque sensible $P$. Dans le second cas, la déviation $u$ obtenue sur la plaque $\mathbf{P}$ correspond à un rayon peu dévié, défini par la source 0 , la fente diaphragme $0^{\prime}$ et le rayon de courbure $R\left(\mathbf{O O}^{\prime}=a ; \mathbf{O}^{\prime} \mathrm{B}=b\right)$. Pour l'appareil à foyer, $z$ croît, sinon proportionnellement à $R, \mathrm{du}$ moins'se rapprochant de cette loi quand $a$ est notablement plus petit que $2 R$. Pour l'appareil à déviation directe, la relation entre $R, u$, $a$ et $b$ équivaut, pour les rayons peu déviés, à la relation $2 R u=b(a+b)$.

On peut d'ailleurs obtenir la distribution exacte des rayons en fonction de la déviation magnétique $z$ ou $u$; pour cela, il suffit d'appliquer la méthode de calcul exposée ci-dessus, mais une courbe de distribution ne sera valable que pour un appareil donné et un champ donné. Pour obtenir ces courbes, on utilise la formule (11) ainsï que les relations suivantes entre $R, z$ et $u$ :

$$
z^{2}=4 R^{2}-a^{2} \quad 4 R^{2} u^{2}=a^{2} u^{2}+\left(x^{2}+a b+b^{2}\right)^{2} .
$$

Les quantités qu'il s'agit d'obtenir sont

$$
P_{z}=P_{s} \frac{\mathrm{d} s}{\mathrm{~d} z}=P_{s} \frac{\mathrm{d} s}{\mathrm{~d} R} \frac{\mathrm{d} R}{\mathrm{~d} z} ; \quad P_{u}=P_{s} \frac{\mathrm{d} s}{\mathrm{~d} u}=P_{s} \frac{\mathrm{d} s}{\mathrm{~d} R} \frac{\mathrm{d} R}{\mathrm{~d} u} .
$$

En effectuant les calculs, on trouve

$$
\begin{aligned}
& P_{z}=\frac{3}{8} \frac{1+2 \alpha}{a^{6}}\left[\alpha^{4}+\left\{(1+2 \alpha)\left(\frac{A}{g}-1\right)-\alpha^{4}\right\}^{2}\right] \frac{H^{2} z}{4 g A}, \text { où } A=\sqrt{g^{2}+\frac{H^{2}}{4}\left(z^{2}+a^{2}\right)} ; \\
& P_{u}=\frac{3}{8} \frac{1+2 \alpha}{\alpha^{6}}\left[\alpha^{4}+\left\{(1+2 \alpha)\left(\frac{B}{2 g u}-1\right)-\alpha^{2}\right\}^{2}\right] \frac{H^{2}\left[\left(a b+b^{2}\right)^{2}-u^{4}\right]}{2 g B u^{2}} \\
& \text { où } \\
& B=\sqrt{4 g^{2} u^{2}+H^{2}\left[a^{2} u^{2}+\left(u^{2}+a b+b^{2}\right)^{2}\right]} \text {. }
\end{aligned}
$$

Les limites sont à déterminer par la condition que les rayons puissent atteindre la plaque. 
Ainsi, pour $z$ comme pour $u$, la distribution observée ne comprendra qu'une partie des rayons produits, de sorte que l'intégrale $\int P_{z} \mathrm{~d} z$ ou $\int P_{u} \mathrm{~d} u$ prise entre ces limites n'est pas égale à 1 , mais à une fraction qu'on peut calculer. Les figures 8 et 9 donnent l'exemple de courbes de distribution construites pour des appareils de type courant, et on voit que ces courbes ont respectivement une similitude générale avec celles des figures ร̆ et 6 .

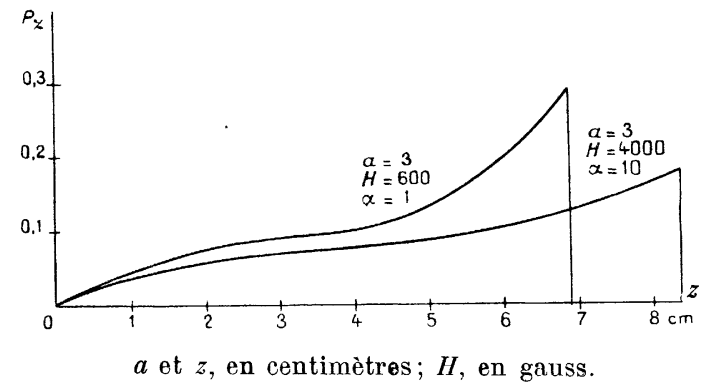

Fig. 8 .

Il semble ainsi que l'emploi du dispositif de déviation directe avec peu de dispersion soit particulièrement favorable pour déceler les rayons $\beta$ provenant de l'effet Compton si la distribution de ceux-ci est conforme à la formule que j'ai admise. En même temps, on est

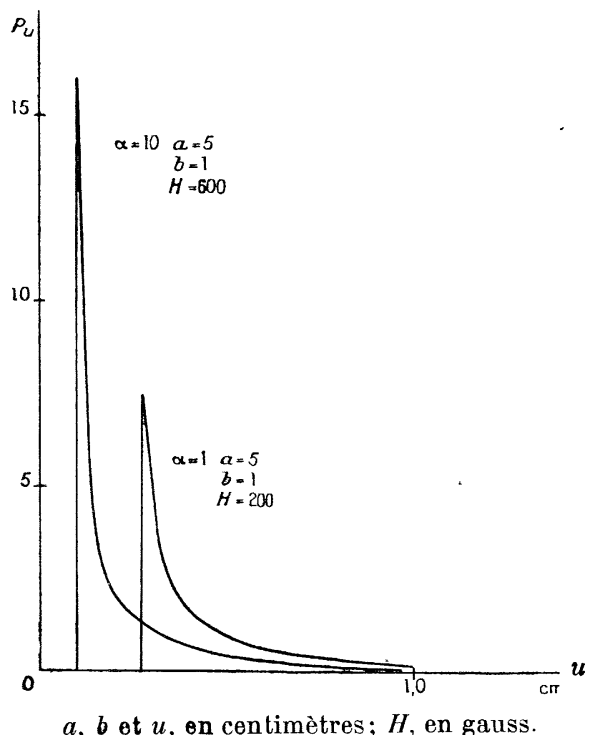

Fig. 9 .

frappé par l'influence que peut avoir le dispositif expérimental sur l'interprétation des spectres magnétiques. En effet, même pour une distribution d'énergie à peu près uniforme, le dispositif de déviation directe doit produire un resserrement de rayons de grande énergie donnant, par contraste. l'impression d'une tète de bande ou peut-être même d'une raie, alors qu'on ne verrait qu'un fond continu à l'appareil à foyer. Ainsi, la définition des raies est plus certaine quand elle a été obtenue ou contròlée à l'aide d'un appareil à foyer.

6. Photoélectrons excités par des rayons $\gamma$ diffusés. - Outre les électrons de choc qui accompagnent les rayons $\gamma$ diffusés, on peut prévoir l'émission par ces derniers de photoélectrons formant une bande limitée avec des énergies qui se déduisent de celles des rayons excitateurs par soustraction d'une énergie de niveau de l'atome excité. 
L'énergie des rayons y diffusés variant de $x m c^{2}$ à $\frac{\alpha}{1+2 x} m c^{2}$, l'énergie des photoélecrons extraits du niveau $i$ variera de $\alpha m c^{2}-w_{i}$ à $\frac{\alpha}{1+2 \alpha} m c^{8}-u_{i}$. La distribution de l'énergie dans une telle bande pourrait être prévue d'après la proportion de quanta de longueur d'onde comprise entre $\lambda$ et $\lambda+d \lambda$ (formule 7) et d'après le coefficient d'absorption. Celui-ci est peu connu dans le cas où il s'agit d'une conversion du quantum dans l'atome émetteur, mais devrait être proportionnel à $\lambda^{3}$ dans le cas de l'absorption normale dans un radiateur secondaire. Il peut y avoir superposition partielle de bandes relatives à des photoélectrons issus des divers niveaux; la bande formée par les électrons de choc peut aussi se superposer à une ou plusieurs bandes de photoélectrons relatifs au même groupe de rayons $\gamma$ primaires diffusés. Quant $\alpha<0,5$, le domaine d'énergie de ces rayons $\gamma$ diffusés (limites $\alpha$ et $\left.\frac{\alpha}{1+2 \alpha}\right)$ est séparé de celui des électrons de choc $\left(\operatorname{limites} \frac{2 \alpha^{2}}{1+2 \alpha}\right.$ et 0 ) et il peut en ètre de mème pour ces derniers et pour les photoélectrons de niveaux superficiels. Pour $\alpha>0,5$, le domaine d'énergie des électrons de choc pénètre dans celui des rayons $\gamma$ diffusés, et il y a nécessairement interpénétration des bandes d'électrons de choc et de photoélectrons, si ces deux phénomènes se produisent avec une importance comparable. Le nombre de photoélectrons de rayons diffuséz ne peut, d'ailleurs, dépasser celui des électrons de choc, et doit plutôt lui être inférieur, puisque tous les quanta diffusés ne sont probablement pas convertis en photoélectrons. Indiquons, à titre d'exemple, que, pour le groupe de rayons $\gamma$ primaires du RaC de 610 kilovolts, le domaine des rayons $\gamma$ diffusés s'étend de 610 à $180 \mathrm{kv}$; celui des électrons de choc, de $\mathbf{4 3 0} \mathbf{k v}$ à $\mathbf{0}$, et celui des photoélectrons extraits du niveau $\mathrm{K}$ du plomb, de 523,4 à $92,4 \mathrm{kv}$.

7. Discussion. - Les considérations ci-dessus exposées peuvent servir de guide pour rechercher les rayons $\gamma$ diffusés sur les spectres de diffraction, et les rayons $\beta$ pouvant résulter de l'effet Compton sur les spectres magnétiques primaires ou secondaires.

En présence d'un spectre dont l'apparence suggérerait une interprétation par effet Compton, on pourrait avoir recours à une exploration photométrique, en tenant compte de la largeur occupée normalement dans ce spectre par les rayons $\beta$ de vitesse déterminée, en raison des dimensions des diaphragmes et de la source. Cependant, on a encore peu de données sur la relation entre le noircissement de la plaque et le nombre de rayons reçus par unité de temps et de surface, ainsi que la vitesse de ces rayons et, à ce point de vue, la plaque sensible pourrait être remplacée par un récepteur permettant de recueillir et de mesurer la charge des rayons. Il peut y avoir superposition de phénomènes résultant de a diffusion de plusieurs groupes monochromatiques de rayons $\gamma$ ou superposition de spactre continu lù à l'effet Compton à un spectre continu indépendant de cet effet. La question apparaît donc d'une grande complexité et demande une étude approfondie.

L'examen général des données jusqu'ici disponibles ne permet pas encore de conclure d'une manière absolument convaincante. Les phénomènes qui paraissent dominants sont les raies dues aux photoélectrons et le fond continu. Dans le spectre magnétique des rayons $\beta$ du $\mathrm{RaE}$, les raies manquent, sauf un faible groupe de grande vitesse ('), et le fond continu, obtenu avec un appareil à déviation directe, affecte la forme d'une large . bande débutant vers 1100 kilovolts, atteignant un maximum vers $\mathbf{4 0 0}$ kilovolts et s'éteignant vers 100 kilovolts. Outre que cette distribution ne présente pas les caractères prévus, il n'est guère possible de l'attribuer à l'effet Compton, puisque les rayons $y$ dont l'énergie devrait être, en ce cas, supérieure à celle des rayons $\beta$ (formule 6) sont à peine représentés dans l'émission du RaE. Les mesures récentes de la charge des rayons de RaB et de $\mathrm{RaG}$ (*) ont fait connaître la distribution du spectre continu pour chacun de ces corps en fonction de $R H$; le nombre total de rayons correspond à l'émission d'un peu plus qu'un

(') Irène Curie et J. d'Espine, C. R., t. 181 (1925), p. 31.

(") Gurney, Proc. Roy. Soc., t. 109 (1925), p. 540. 
électron par atome détruit, l'excès étant 10 p. 100 à 25 p. 100; il est donc possible que ce spectre continu comprenne les électrons primaires accompagnés de photoélectrons et d'électrons de choc. Quant aux régions de très grande vitesse $(R H>10000 \mathrm{U}$. E . M. $)$, où l'existence de radiations $\beta$ avait été signalée par Danysz, par Rutherford et Robinson (1) et qui ont été récemment explorées par d'Espine et Yovanovitch ( ${ }^{2}$, les intensités y sont si faibles qu'on ne peut encore dire s'il s'agit de raies ou de têtes de bande pouvant correspondre à l'effet Compton. Il existe cependant, dans ces régions, un fond continu avec les apparences de lètes de bandes.

Dans son récent examen du spectre des rayons $\beta$ de RaAc et de AcX, L. Meitner attire l'attention sur ce fait que ces substances, qui se transforment avec émission de rayons $\alpha$, donnent, en plus de raies, un spectre continu qui ne doit pas contenir d'électrons primaires, mais pourrait s'expliquer par l'effet Compton $\left(^{3}\right)$.

J. Thibaud a signalé, dans les spectres magnétiques d'origine secondaire, la présence d'un fond continu qui débute aux raies de nature photoélectrique et leur donne un prolongement vers les vitesses faibles $\left.{ }^{4}\right)$. Il en cherche l'interprétation dans le phénomène Compton en faisant intervenir l'effet photoélectrique des rayons $\gamma$ diffusés. Rappelons qu'à la bande de cette provenance, devrait se superposer une bande comprenant les électrons de choc et débutant à une certaine distance de la raie. Par exemple, pour le groupe primaire de rayons $\gamma$ de $\mathrm{RaC}$ de $610 \mathrm{kv}(a==1,2)$, le domaine des rayons diffusés par effet Compton est compris entre 610 et $180 \mathrm{kv}$; celui des photoélectrons extraits du niveau $\mathrm{K} \mathrm{du}$ plomb, entre 523,4 et $92,4 \mathrm{kv}$; celui des électrons de choc, entre $430 \mathrm{kv}$ et 0 . La proportion de photoélectrons relative aux rayons diffusés dont la longueur d'onde est comprise entre $\lambda$ et $\lambda+\mathrm{d} \lambda$, devrait être proportionnelle à $\lambda^{3} P_{\lambda}$, et l'énergie de ces électrons, comprise entre

$$
\frac{h c}{\lambda}-w_{\mathrm{K}} \quad \text { et } \quad \frac{h c}{\grave{\lambda}}-\frac{h c}{\lambda^{2}} \mathrm{~d} \lambda-w_{\mathrm{K}},
$$

serait répartie sur un intervalle proportionnel à $d \lambda / \lambda^{2}$, de sorte que la densité de distribution en fonction de l'énergie serait proportionnelle à $\lambda^{5} P_{\lambda}$ et pourrait être calculée d'après (7). Pour $a=1(509 \mathrm{kv})$, ceci conduit à une distribution d'abord à peu près uniforme au voisinage immédiat de la raie, puis la densité s'accroît de plus en plus rapidement vers les vitesses plus faibles. Il n'y a pas de contradiction entre ces prévisions et l'aspect des clichés; toutefois, la comparaison avec la théorie est rendue difficilè du fait que le radiateur n'entoure pas la source et que son épaisseur est assez grande pour que la distribution des rayons $\beta$ et $\gamma$ provenant de ses couches plus profondes soit modifiée en traversant les couches plus superficielles.

Note ajoutée à la correction. - Il convient de signaler un travail qui vient de paraître sur le spectre des rayons $\beta$ de RaD [Curtiss, Phys. Rev., t. 27 (1926), p. 23̈7, Ce corps émet un seul groupe de rayons $\gamma$ primaires auquel correspondent 5 raies du spectre magnétique dues aux photoélectrons issus des niveaux électroniques de l'atome transformé. Les plus fortes de ces raies sont des têtes de bandes se prolongeant vers les vitesses faibles; leur interprétation par effet Compton pourrait être tentée, bien que, dans ce cas comme dans d'autres, la distribution des vitesses des électrons primaires émis lors de la transformation radioactive n'ait encore pu ètre précisée.

(1) Darysz, These de Doctorat, Paris (1913). - Ruthrnpond et Robinson, Phil. Mag., t. 26 (1913), p. 717.

(2) D'Espine et Yovanovitgh, C. R., t. 178 (1924), p. 1811; t. 179 (1924), p. 1162; t. 180 (1924). p. 202. d'Espiss, $C . R$, t. 180 (1925), p. 1403.

(3) L. Meitrer, Zts. $t$. Phys., t. 34 (1925), p. 807.

(4) J. Thibadd, Thèse de Doctorat, J. Phys., t. 6(1925), p. 82 et 334.

Manuscrit reçu le 26 février 1926. 